\title{
DECOMPOSIÇÃO DE ESPÉCIES UTILIZADAS COMO ADUBAÇÃO VERDE EM SISTEMA AGROFLORESTAL EXPERIMENTAL, SANTARÉM, PARÁ
}

\author{
lara Rayana Leal de Sousa'; Daniela Pauletto²; Lucas Sérgio de Sousa Lopes³ Rafael Rode4. \\ 1 Universidade Federal do Oeste do Pará, Santarém, Pará, Brasil, rayana.iaraleal@gmail.com \\ 2 Universidade Federal do Oeste do Pará, Santarém, Pará, Brasil, danielapauletto@hotmail.com \\ ${ }^{3}$ Engenheiro Florestal, Santarém, Pará, Brasil, lucaasergio@gmail.com \\ ${ }^{4}$ Universidade Federal do Oeste do Pará, Santarém, Pará, Brasil, rafaelrode@gmail.com
}

RESUMO: Os sistemas agroflorestais são consórcios que possuem em seus arranjos culturas alimentares e madeireiras, tendo sua sustentabilidade estabelecida em função das interações do fluxo de decomposição das espécies existentes. Nesse sentido, a adubação verde é a nova prática utilizada para promover a ciclagem de nutrientes nos sistemas de produção, sendo as leguminosas as espécies mais indicadas para compor esse tipo de adubação. Dessa forma, este trabalho teve por objetivo avaliar a taxa de decomposição foliar das espécies feijão de porco (Canavalia ensiformis (L.) DC.), gliricídia (Gliricidia sepium (Jacq.) Walp.), guandú (Cajanus cajan L.) e leucena (Leucaena leucocephala (Lam.) de Wit.), utilizadas como adubação verde em sistema agroflorestal (SAF) adotando-se do método de litter bags. O estudo foi realizado no SAF experimental, Universidade Federal do Oeste do Pará (UFOPA) no período de abril a setembro de 2017. Analisou-se a decomposição da fração foliar durante 5 períodos de tempo, com coletas aos 30, 60, 90, 120 e 150 dias. Os dados gerados foram ajustados por meio de equação não-linear para obtenção da constante de decomposição (k) e cálculo do tempo de meia vida, sendo analisados estatísticamente pelo teste de Tukey a 5\% de probabilidade. A gliricídia apresentou maior taxa de decomposição em relação às demais espécies avaliadas, contudo o guandú embora tenha obtido taxa mais lenta, pode auxiliar na manutenção da cobertura e umidade do solo. A decomposição de todas as espécies se mostrou mais acentuada durante os 90 primeiros dias de experimento.

PALAVRAS-CHAVE: Biomassa, Decomposição foliar, Leguminosa.

\section{DECOMPOSITION OF SPECIES USED AS GREEN MANURE IN AN EXPERIMENTAL AGROFORESTRY SYSTEM, SANTARÉM, PARÁ}

ABSTRACT: Agroforestry systems are consortia that have food and timber crops in their arrangements, and their sustainability is established as a function of the interactions of the decomposition flow of the existing species. In this sense, green manuring is the new practice used to promote nutrient cycling in production systems, with legumes being the most suitable species to compose this type of fertilization. The objective of this work was to evaluate the leaf decomposition rate of the bean 
(Canavalia ensiformis (L.) DC.), gliricidia (Gliricidia sepium (Jacq.) Walp.), pigeon pea (Cajanus cajan L.) and leucaena (Leucaena leucocephala (Lam.) de Wit.) used as green fertilizer in an agroforestry system (SAF) using the litter bags method. The study was carried out in the experimental SAF, Federal University of the West of Pará (UFOPA) from april to september 2017. The leaf fraction was analyzed for 5 times periods, with collections at 30,60, 90, 120 and 150 days. The data were adjusted by means of a nonlinear equation to obtain the decomposition constant $(k)$ and the calculation of the half-life time, being analyzed statistically by the Tukey test at 5\% probability. The gliricid. Presented a higher decomposition rate in relation to the other species evaluated, but the guandu, although it obtained a slower rate, could help to maintain the cover and soil moisture. The decomposition of all species was more pronounced during the first 90 days of the experiment.

KEYWORDS: Biomass, Leaf decomposition, Leguminous.

\section{DESCOMPOSICIÓN DE ESPECIES UTILIZADAS COMO FERTILIZACIÓN VERDE EN SISTEMA AGROFORESTAL EXPERIMENTAL, SANTARÉM-PA}

RESUMEN: Los sistemas agroforestales son consorcios que poseen en sus arreglos cultivos alimentarios y madereros, teniendo su sostenibilidad establecida en función de las interacciones del flujo de descomposición de las especies existentes. En este sentido, la fertilización verde es la nueva práctica utilizada para promover el ciclo de nutrientes en los sistemas de producción, siendo las leguminosas las especies más indicadas para componer ese tipo de fertilización. En el presente estudio se evaluó la tasa de descomposición foliar de las especies de frijol de cerdo (Canavalia ensiformis (L.) DC.), gliricardia (Gliricidia sepium (Jacq.) Walp.), guandú (Cajanus cajan L.) y leucena (Leucaena leucocephala (Lam.) de Wit.), utilizadas como fertilización verde en sistema agroforestal (SAF) adoptando el método de litter bags. El estudio fue realizado en el SAF experimental, Universidad Federal del Oeste de Pará (UFOPA) en el período de abril a septiembre de 2017. Se analizó la descomposición de la fracción foliar durante 5 períodos de tiempo, con colectas a los 30, 60, 90, 120 y 150 días. Los datos generados fueron ajustados por medio de ecuación no lineal para obtener la constante de descomposición (k) y cálculo del tiempo de media vida, siendo analizados estadísticamente por la prueba de Tukey al 5\% de probabilidad. La gliricídia presentó mayor tasa de descomposición en relación a las demás especies evaluadas, sin embargo, el guandú aunque obtuvo una tasa más lenta, puede auxiliar en el mantenimiento de la cobertura y humedad del suelo. La descomposición de todas las especies se mostró más acentuada durante los 90 primeros días de experimento.

PALABRAS-CLAVE: Biomasa, Descomposición foliar, Legumbres. 


\section{INTRODUÇÃO}

Os sistemas agroflorestais - SAFs são sistemas de produção que consorciam espécies florestais (frutíferas e/ou madeireiras) com cultivos agrícolas e em alguns casos também animais, na mesma área e numa sequência temporal (PALUDO; COSTABEBER, 2012). O uso destes sistemas integrados está se inserindo como uma nova alternativa de produção para pequenos e médios agricultores. Macêdo et al. (2001), expõe que a vantagem desse tipo de sistema, é que o mesmo permite um fluxo de renda constante, desde os primeiros anos de implantação e por longo período.

Rodrigues (2004) evidenciou estes sistemas possuem sustentabilidade própria, que ocorrem em função das interações do fluxo de energia, da ciclagem de nutrientes e da biodiversidade do sistema. Com isso, os nutrientes principais são conseguidos através da decomposição da biomassa, e são associados com os tecidos das plantas, tornando os nutrientes prontamente disponíveis para culturas comerciais (SILVA et al., 2008). As plantas incorporam carbono (C) através do processo de fotossíntese, formando a biomassa vegetal, durante o seu crescimento parte dessa biomassa produzida retorna ao solo, formando a camada de serrapilheira (COSTA et al., 2004). A ação do processo de decomposição sobre a serrapilheira proporciona então a ciclagem de nutrientes.

Segundo Cardoso et al. (2014), adubação verde é uma prática que consiste no uso de espécies vegetais em rotação, sucessão ou consorciação com outras culturas comerciais, objetivando melhoria, manutenção e recuperação das propriedades físicas, químicas e biológicas do solo. $\bigcirc$ uso dessas plantas aumenta a sustentabilidade dos sistemas em que as mesmas estão inseridas, pelo fato de possuírem a capacidade de absorver nutrientes presentes nas camadas subsuperficiais do solo e liberandos pela decomposição dos seus resíduos (BERNARDES et al., 2010).

De acordo com Leite et al. (2010) e Giongo et al. (2011), estas espécies possuem capacidade de gerar matéria 
seca (MS) suficientes para manter o solo coberto, aumentando assim o teor de matéria orgânica e diminuindo a evapotranspiração. Por este motivo, atualmente, espécies "adubadeiras" estão sendo inseridas nos arranjos de SAF's em razão de sua alta capacidade de fixar nitrogênio e de produzir biomassa (SILVA et al., 2014). Além disso, melhoram a fertilidade do solo, propiciando o aumento da produtividade (PAULA et al., 2015).

Por apresenta maior aporte nutricional, a fração foliar, segundo Cabianchi (2010), é considerada o componente principal da serapilheira depositada no solo pelas espécies arbóreas. Dessa forma, este trabalho teve por objetivo avaliar a taxa de decomposição foliar de quatro espécies, sendo duas arbóreas, uma arbustiva e uma herbácea, utilizadas como adubação verde adotando-se o uso de litter bags em sistema agroflorestal experimental, no município de Santarém $-P A$.

\section{MATERIAL E MÉTODOS}

O estudo foi realizado no sistema de produção agroflorestal experimental, inserido na Unidade Experimental de Campo (UEC) da Universidade Federal do Oeste do Pará (UFOPA), situada no Km 37 da Rodovia Santarém/Curuá-Una - PA 370 (020 24" 52"S e 54 42" 36"W), no município de Santarém, região oeste do Estado do Pará. Este sistema foi implantado em março de 2016, e possui 1 ha ${ }^{-1}$ de plantio consorciado.

A região apresenta clima tipo quente e úmido segundo a classificação de Köppen, com chuvas concentradas no primeiro semestre do ano (figura 1), com temperatura média anual variando de $25^{\circ}$ a $28^{\circ} \mathrm{C}$, com umidade relativa média do ar de 86\%. A precipitação pluvial média anual é de 1920 mm variando de $170 \mathrm{~mm} \cdot \mathrm{mês}^{-1}$ a $60 \mathrm{~mm} \cdot \mathrm{mês}^{-}$ 1'(SANTARÉM，2008; FERREIRA， 2011) (Figura 1). Segundo IDESP (2014) Os solos são considerados do tipo Latossolo Amarelo de texturas médias, argilosas e muito argilosas. 
Figura 1. Dados de precipitação pluviométrica mensais (janeiro de 2017 a setembro de 2017), extraídos do pluviômetro instalado na Fazenda Experimental da Universidade Federal do Oeste do Pará.

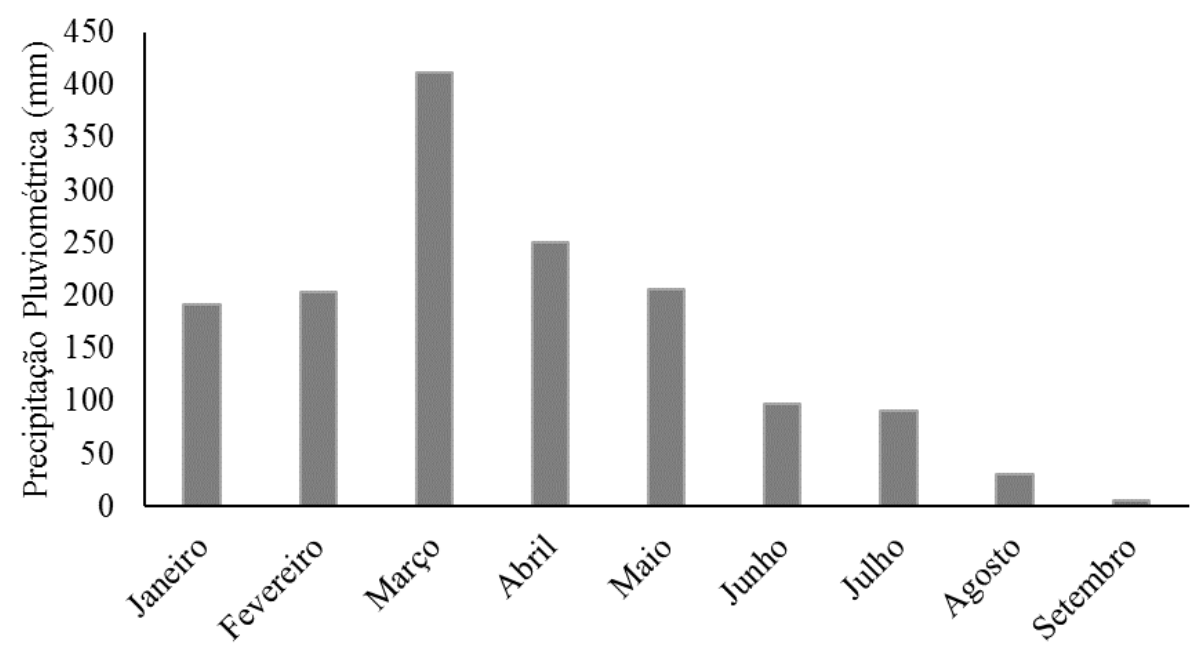

Meses/ 2017

O estudo foi conduzido entre os foliar de quatro espécies utilizadas meses de abril e setembro de 2017. como componentes de adubação Foram coletadas amostras da fração verde (Tabela 1).

Tabela 1. Espécies utilizadas como adubação verde e estágio fenológico no momento da coleta das folhas.

\begin{tabular}{llcc}
\hline Nome popular & \multicolumn{1}{c}{ Nome Científico } & $\begin{array}{c}\text { Tipo de } \\
\text { Vegetação }\end{array}$ & Estágio fenológico \\
\hline Feijão de Porco & Canavalia ensiformis (L.) DC. & Herbácea & Frutificação \\
Gliricídia & Gliricidia sepium (Jacq.) Walp. & Arbórea & Vegetativo \\
Guandú & Cajanus cajan L. & Arbustiva & Vegetativo \\
Leucena & Leucaena leucocephala (Lam.) de Wit.) & Arbórea & Frutificação \\
\hline
\end{tabular}


material foliar foi seco ao ar por cinco dias, com o intuito de diminuir o excesso de umidade, permitindo a obtenção de massa seca inicial sem interferir com o uso de secagem artificial do material. Tanner (1981) e Scheer (2008) corroboram que a présecagem em estufa do material antes da instalação do experimento de decomposição pode intervir em resultados de taxa de decomposição mais lenta nos meses iniciais de coleta. Para estimativa de decomposição, porções de $20 \mathrm{~g}$ de folhas foram pesadas e acondicionadas em bolsas de náilon (litter bags), de malha 2 mm e dimensões de $20 \times 20$ cm, conforme metodologia preconizada por Scheer (2008) e Silva et al. (2014). A malha utilizada teve o propósito de permitir o acesso da mesofauna, bem como microorganismos do solo que estão inseridos no processo de decomposição natural de folhas.

Os litter bags foram distribuídos de forma aleatória sobre a superfície do solo do sistema agroflorestal, simulando a queda natural do material formador de serapilheira (FERNANDES et al., 2006). As coletas foram estabelecidas aos 30, 60, 90, 120 e 150 dias, onde a cada período eram retiradas quatro bolsas por espécie avaliada. Após coletado, o material foliar contido em cada bolsa de decomposição foi triado manualmente, para retirada de partículas de solos e, em seguida submetido à secagem em estufa a $65^{\circ} \mathrm{C}$ até obtenção de massa constante (SCORIZA et al., 2012).

Os coeficientes de decomposição (k) da fração foliar de cada espécie estudada foram obtidos a partir dos valores de massa remanescentes ao longo dos cinco meses de estudo, os quais foram ajustados ao modelo exponencial proposto por Thomas \& Asakawa (1993), empregando-se o programa STATISTICA V. 10.

$$
P_{t}=P_{0} \cdot e^{-k t}
$$

Onde, $\mathrm{P}_{\mathrm{t}}=$ peso seco remanescente $\mathrm{da}$ amostra após $\mathrm{t}$ dias; $\mathrm{P}_{0}=$ peso seco inicial, colocado nos sacos no tempo zero $(t=0) ; t=$ tempo em dias e $k=$ constante de decomposição. 
Calculou-se ainda, a partir da constante de decomposição (k), o tempo de meia vida das folhas, proposto por Landsberg e Gower (1997). Este valor é obtido por meio da linearização do modelo anterior, e seu resultado corresponde ao tempo requerido para que ocorra a transformação de metade da quantidade inicial do material foliar analisado. Desta forma adotou-se a equação:

$$
t_{1 / 2}=\ln (2) / k
$$

onde $t_{1 / 2}=$ tempo de meia vida das folhas e $\mathrm{k}=$ constante de decomposição obtida a partir do ajuste do modelo não linear.

Os dados de decomposição da fração foliar (massa remanescente a cada mês) foram submetidos à análise de variância, com delineamento inteiramente casualizado, com quatro repetições, por meio do programa estatístico ASSISTAT $\vee .7 .7$ beta. Adotou-se o teste Tukey a 5\% de significância para comparação de médias.

\section{RESULTADOS E DISCUSSÃO}

Durante os 150 dias de avaliação da decomposição foliar nos litter bags, a perda de massa da fração foliar foi menor para a espécie guandú, segundo o teste Tukey (Tabela 2).

Observou-se que as espécies feijão de porco e gliricídia perderam mais $50 \%$ de sua massa inicial, não apresentando diferença estatística entre si durante todo o experimento.

O guandú, foi a espécie que menos perdeu massa e essa tendência foi constatada durante os cinco meses de experimento. Observa-se que aos 30 dias, aproximadamente $12 \%$ de massa foliar de guandú ainda permanecia nos litter bags. Salmi et al. (2006), ao avaliar a decomposição de fitomassa de guandú, apresenta valores similares ao verificar que no mesmo período de tempo (30 dias), 75\% da fitomassa ainda restava sobre o solo. Dessa forma, o mesmo autor infere que o uso dessa espécie inserida a um sistema integrado, acarreta em uma boa cobertura do solo, obtida tanto por meio da queda natural das folhas, 
quanto por meio de poda da espécie, favorecendo ainda a conservação da umidade do solo e o controle de plantas daninhas.

Tabela 2. Decomposição mensal da fração foliar de espécies utilizadas na adubação verde.

\begin{tabular}{lccccc}
\hline Espécies adubadeiras & Maio & Junho & Julho & Agosto & Setembro \\
\hline Feijão de Porco & $7,20 \mathrm{c}$ & $4,88 \mathrm{bc}$ & $3,12 \mathrm{~b}$ & $3,39 \mathrm{~b}$ & $4,90 \mathrm{a}$ \\
Gliricídia & $6,79 \mathrm{c}$ & $3,67 \mathrm{c}$ & $3,93 \mathrm{~b}$ & $2,91 \mathrm{~b}$ & $3,25 \mathrm{a}$ \\
Guandú & $12,21 \mathrm{a}$ & $9,44 \mathrm{a}$ & $9,59 \mathrm{a}$ & $7,67 \mathrm{a}$ & $6,96 \mathrm{a}$ \\
Leucena & $10,29 \mathrm{~b}$ & $6,78 \mathrm{ab}$ & $4,0 \mathrm{~b}$ & $4,04 \mathrm{ab}$ & $3,69 \mathrm{a}$ \\
\hline Médias seguidas da mesma letra, na coluna, não diferem estatisticamente entre si pelo teste de Tukey
\end{tabular}
a 5\% de significância.

Ao analisarmos a Tabela 3, a mesma apresenta os coeficientes de decomposição para as espécies em estudo, bem como, seus tempos de meia-vida. Observa-se que a espécie gliricídia obteve o maior coeficiente de decomposição (k) entre as adubações verdes analisadas. O tempo estimado para que ocorra a decomposição de 50 \% da fração foliar (t/1/2) variou entre 26,23 dias (gliricídia) e 73,15 dias (guandú), seguindo a mesma tendência do coeficiente ( $k$ ). No caso da gliricídia, Silva et al. (2007) apontaram valores aproximados aos encontrados neste estudo ao avaliar a decomposição foliar utilizando a metodologia de litter bags para essa espécie, onde a mesma apresentou a constante de decomposição de 0,003 g.g ${ }^{-1}$ dia e $t_{1 / 2}$ de 21 dias, considerando que os resultados foram obtidos durante meses mais chuvosos.

Contrapondo-se a isto, Souza et al. (2010), obtiveram resultados divergentes ao avaliar os parâmetros de decomposição para gliricídia, apresentando $k=0,0077 \mathrm{~g} \cdot \mathrm{g}^{-1} \mathrm{dia}$ e tempo de meia vida de 90 dias. Porém, a decomposição da espécie guandú possui tendência similar/ aproximada aos resultados obtidos neste trabalho, sendo a constante de decomposição (k) igual a 0,0070 g.g $\mathrm{g}^{-1}$ dia e $\mathrm{t}_{1 / 2}$ de 99 dias. 
Tabela 3. Coeficientes de decomposição, obtidos por ajuste de modelo exponencial e tempo de meia vida da fração foliar da serapilheira.

\begin{tabular}{lccccc}
\hline Espécies & \multicolumn{5}{c}{ Parâmetros } \\
\cline { 2 - 6 } adubadeiras & $\mathrm{P}_{\mathrm{o}}(\mathrm{g})$ & $\mathrm{K}\left(\mathrm{g} \cdot \mathrm{g}^{-1} \mathrm{dia}\right)$ & $\mathrm{r}^{2}$ & $\mathrm{EPE}$ & $\mathrm{t}_{1 / 2}$ (dias) \\
\hline Feijão de Porco & 20 & 0,0244 & 0,89 & 0,0020 & 28,40 \\
Gliricídia & 20 & 0,0264 & 0,94 & 0,0025 & 26,23 \\
Guandú & 20 & 0,0095 & 0,90 & 0,0007 & 73,15 \\
Leucena & 20 & 0,0165 & 0,93 & 0,0013 & 41,91 \\
\hline Po:
\end{tabular}

Po: massa inicial; $k$ : constante da decomposição; $r^{2}$ : coeficiente de determinação do ajuste para a estimativa do k; EPE: Erro-padrão da estimativa de k; $t_{1 / 2}$ : tempo de meia vida do material foliar; ${ }^{\star} p<0,001 .{ }^{* \star} p<0,0001$.

Contudo, apesar de a gliricídia haver apresentado taxa de decomposição alta, isso não justifica que a decomposição mais lenta do guandú interfira negativamente na escolha da espécie para compor o arranjo de um sistema agroflorestal. Por outro lado, o valor mais baixo da decomposição apresentado por essa espécie pode ser interessante, isso depende da cultura a ser consorciada com essa leguminosa (PAULA et al., 2015), uma vez que a liberação da carga de nutrientes mediante a decomposição foliar está ocorrendo de forma lenta, esta pode favorecer o desenvolvimento da cultura componente do arranjo agroflorestal à médio e longo prazo.

Na Figura 2, estão representadas as curvas que refletem o padrão de decomposição da fração foliar das quatro espécies em estudo. Estas curvas foram obtidas por meio do ajuste do modelo exponencial nãolinear que obtiveram coeficientes de determinação $\left(r^{2}\right)$ variando entre 0,89 e 0,94 (Tabela 3).

Para todas as espécies, as curvas de ajuste do processo de decomposição dos resíduos foliares apresentaram padrão semelhante, onde nos três meses iniciais do experimento a decomposição pela perda de massa foliar é mais rápida, seguido uma fase mais lenta que tende à estabilizar a curva. Esse mesmo desenho gráfico é exposto por Souza et al. (2010) e Rossi (2013), ao avaliarem a taxa de decomposição de espécies leguminosas com potencial de 
adubação verde nos estados do Rio de Janeiro e Goiás.

O fato de a decomposição ter sido mais acentuada durante os 90 primeiros dias do experimento está relacionada, provavelmente, à presença de chuvas ou precipitação considerável nesse período (Figura 1).
De acordo com Alves et al. (2006) e Menezes et al. (2010) a velocidade de decomposição está relacionada às condições favoráveis de conteúdo de água no solo, estando, portanto, ligada aos índices de precipitação de cada local.

Figura 2. Curvas de decomposição da fração foliar da serapilheira ajustadas ao modelo exponencial simples para obtenção das constantes de decomposição (k).

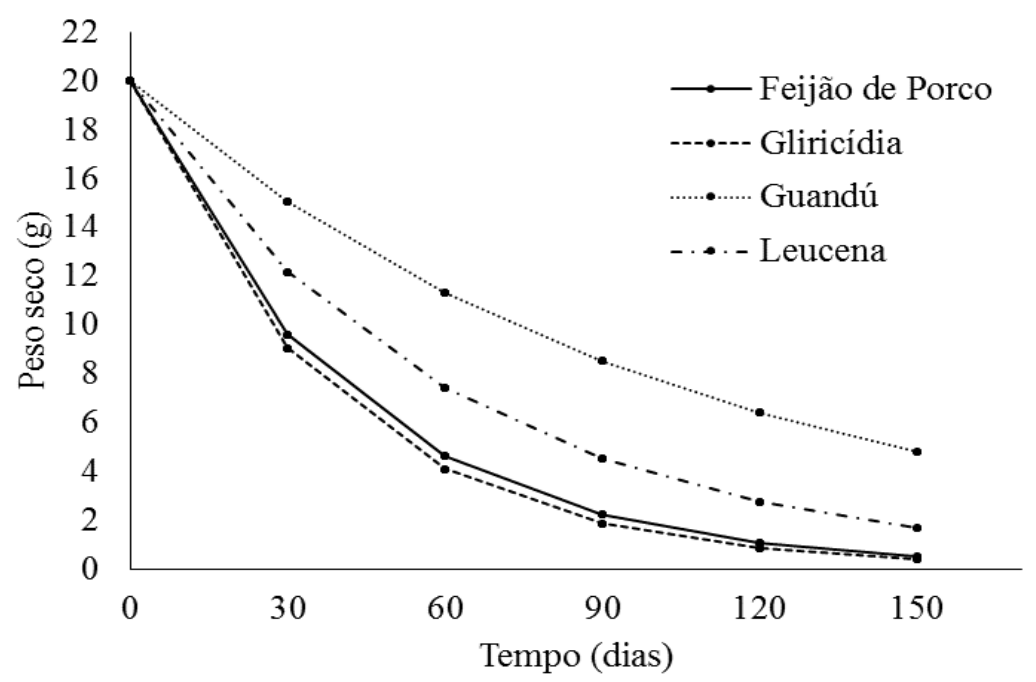

\section{CONCLUSÃO}

A espécie gliricídia apresentou maior taxa de decomposição da fração foliar, com perda de $75,5 \%$ de sua massa foliar durante os 150 dias avaliados. A espécie mostra vantagens para uso em sistemas consorciados por possuir capacidade de incorporar nutrientes ao solo de forma mais rápida em relação às outras espécies estudadas.

O feijão de porco, obteve resultados de decomposição aproximados aos da espécie gliricídia. 
Apesar do guandú, leucena e feijão de porco possuírem decomposição mais lenta em relação à gliricídia, o que determina seu uso é a finalidade proposta pelo arranjo de SAF em que a mesma vai ser inserida.

A taxa de decomposição obteve resultados maiores no período de 0 a 90 dias, esses valores de acordo com a literatura, estão relacionados com o índice pluviométrico e a umidade do solo no período de contato dessas espécies com o solo.

\section{AGRADECIMENTOS}

Ao Grupo de Pesquisa Centro de Estudos em Manejo e Sistemas Florestais Integrados, pelo apoio financeiro para o desenvolvimento dessa pesquisa.

\section{REFERÊNCIAS}

ALVES, A. R.; SOUTO, J. S.; SOUTO, P. C.; HOLANDA, A. C. Aporte e decomposição de serrapilheira em área de Caatinga, na Paraíba. Revista de Biologia e Ciências da Terra, v. 2, 2006.

BERNARDES, T. G.; SILVEIRA, P. M.; MESQUITA, M. A. M.; AGUIAR, R. A.; MESQUITA, G. M. Decomposição da biomassa e liberação de nutrientes dos capins braquiária e mombaça, em condições de cerrado. Pesquisa Agropecuária Tropical, v. 40, p. 370377, 2010.

CABIANCHI, G. M. Ciclagem de nutrientes via serapilheira em um fragmento ciliar do rio Urupá, Rondônia, Piracicaba, 2010. 101 f. Dissertação (Mestrado - Programa de Pós-graduação em Ciências) CENA/USP, Piracicaba, 2010.

CALEGARI, A. Perspectivas e estratégias para a sustentabilidade e o aumento da biodiversidade dos sistemas agrícolas com o uso de adubos verdes. In: FILHO, O. F. de L.; AMBROSANO, E. J.; ROSSI, F; CARLOS, J. A. D. Adubação verde e plantas de cobertura no Brasil. Fundamentos e Práticas - Volume I. Brasília - DF: EMBRAPA, 2014, p 19-36.

CARDOSO, R. A.; BENTO, A. S.; MORESKI, H. M.; GASPAROTTO, F. Influência da adubação verde nas propriedades físicas e biológicas do solo e na produtividade da cultura de soja. Semina: Ciências Biológicas e da Saúde, v. 35, p. 51-60, 2014

COSTA, G.S.; FRANCO, A.A.; DAMANESCENO R.N.; FARIA, S.M. Aporte de nutrientes pela serrapilheira em uma área degradada revegetada com leguminosas arbóreas. Revista Brasileira de Ciência do Solo, v.28, p. 919-927, 2004.

FERNANDES, M. M.; PEREIRA, M. G.; MAGALHÃES, L. M. S.; CRUZ, A. R.; 
GIÁCOMO, R. G. Aporte e decomposição de serapilheira em áreas de floresta secundária, plantio de sabiá (Mimosa caesalpiniaefolia Benth.) e andiroba (Carapa guianensis Aubl.) na FLONA Mário Xavier, RJ. Ciência Florestal, v. 16, p. 163-175, 2006.

FERREIRA, J. D. Análise do plano processo na urbanização de cidades do Baixo Amazonas: o caso de Santarém - Brasil, Belém, 2001. 118 f. Dissertação (Mestrado em Desenvolvimento e Meio Ambiente Urbano) - Universidade da Amazônia, Belém, 2011.

GIONGO, $V_{\text {.; }}$ MENDES, A. M. S.; CUNHA, T. J. F.; GALVÃO, S. R. S. Decomposição e liberação de nutrientes de coquetéis vegetais para utilização no semiárido brasileiro. Revista Ciência Agronômica, v. 42, p. 611- 618, 2011.

G. P. SAlMI; SALMI, A. P.; ABBOUd, A. C. S. Dinâmica de decomposição e liberação de nutrientes de genótipos de guandu sob cultivo em aléias.

Pesquisa Agropecuária Brasileira, v.41, p.673-678, 2006.

IDESP. Estatísticas Municipal de Santarém. 2014. Disponível em: <http://www.idesp.pa.gov.br/pdf/statis ticaMunicipal> Acesso em: 14/03/2015.

LANDSBERG, J.J.; GOWER, S.T. Applications of physiological ecology to forest management. Academic Press, 1997.
LEITE, L. F. C.; FREITAS, R. C. A.; SAGRILO, E.; GALVÃO, S. R. S. Decomposição e liberação de nutrientes de resíduos vegetais depositados sobre Latossolo Amarelo no cerrado maranhense. Revista Ciência Agronômica, v. 41, p. 29-35, 2010.

MACÊDO, J. L. V., WANDELLI, E. V.; SILVA JÚNIOR, J. P. Sistemas agroflorestais: manejando a biodiversidade e compondo a paisagem rural. In: III CONGRESSO BRASILEIRO DE SISTEMAS AGROFLORESTAIS: manejando a biodioversidade e compondo a paisagem rural. Manaus-AM. Documento 17 Embrapa Amazônia Ocidental. p. 13-16. 2001.

MENEZES, C. E. G.; PEREIRA, M. G.; CORREIA, M. E. F.; ANJOS, L. H. C.; PAULA, R. R.; SOUZA, M. E. Aporte e decomposição da serapilheira e produção de biomassa radicular em florestas com diferentes estágios sucessionais em Pinheiral, RJ. Ciência Florestal, v. 20, p. 439-452, 2010.

PALUDO, R.; COSTABEBER, J. A. Sistemas agroflorestais como estratégia de desenvolvimento rural em diferentes biomas brasileiros. Revista Brasileira de Agroecologia, v. 7, n. 2, 2012.

PAULA, P. D.; CAMPELLO, E. F. C.; GUERRA, J. G. M.; SANTOS, G. A.; RESENDE, A. S. Decomposição das podas das leguminosas arbóreas gliricidia sepium e acácia angustissima 
em um sistema agroflorestal. Ciência Florestal, v. 25, p. 791-800, 2015.

RODRIGUES, A.C.G. Ciclagem de nutrientes em sistemas agroflorestais na região tropical: Funcionalidade e Sustentabilidade. In: MULLER, M.W.; RODRIGUES, A.C.G.; BRANDÃO, I.C.F.L.; SERÓDIO, M.H.C.F. Sistemas agroflorestais, tendência da agricultura ecológica nos trópicos: sustento da vida e sustento de vida. Ilhéus - BA: SBSAF/CEPLAC/UENF, 2004, p. 67- 88.

ROSSI, C. Q.; PEREIRA, M. G.; GIÁCOMO, S. G.; BETTA, M.; POLIDORO, J. C. Decomposição e liberação de nutrientes da palhada de braquiária, sorgo e soja em áreas de plantio direto no cerrado goiano. Semina: Ciências Agrárias, v. 34, p. 1523-1534, 2013.

SANTARÉM. Informações Municipais.

SEMPLAM/CIAM, p.30, 2008.

SCHEER, M. B. Decomposição e liberação de nutrientes da serapilheira foliar em um trecho de floresta ombrófila densa aluvial em regeneração, Guaraqueçaba (PR).

Revista Floresta, v. 38, n. 2, 2008.

SCORIZA, R. N.; PEREIRA, M. G.; PEREIRA, G. H. A.; MACHADO, D. L.; SILVA, E. M. R. Métodos para coleta e análise de serrapilheira aplicados à ciclagem de nutrientes. Floresta e Ambiente, v. 2, p. 1-18, 2012.

SILVA, G. T. A.; OLIVEIRA, W. R. D.; MATOS, L. V.; NÓBREGA, P. O.; KRAINOVIC, P. M.; CAMPELLO, E. F. C.;
FRANCO, A. A.; RESENDE, A. S. Boletim de Pesquisa e Desenvolvimento, EMBRAPA Agrobiologia, p. 51, 2007.

SILVA, G. T. A.; MATOS, L. V.; NÓBREGA, P. de O.; CAMPELLO, E. F. C.; RESENDE, A. S. de. Chemical composition and decomposition rate of plants used as green manure. Scientia Agricola, v. 65, n. 3, p. 298-305, 2008.

SILVA, H. F.; BARRETO, A. B.; SOUSA, G. T. O.; AZEVEDO, G. B.; GAMARODRIGUES, E. F.; OLIVEIRA, F. G. R. B. Decomposição de serapilheira foliar em três sistemas florestais no Sudoeste da Bahia. Revista Brasileira de Biociências, v. 12, p. 164-172, 2014.

SILVA, E. C.; AMBROSANO, E. J.; SCIVITTARO, W. B.; MURAOKA, T.; BUZETTI, S.; CARVALHO, A. M. Adubação verde como fonte de nutrientes às culturas. In: FILHO, O. F. de L.; AMBROSANO, E. J.; ROSSI, F; CARLOS, J. A. D. Adubação verde e plantas de cobertura no Brasil. Fundamentos e Práticas - Volume I. Brasília - DF: EMBRAPA, 2014, p 265306.

SOUZA, A. P.; LIMA, M. E.; CARVALHO, D. F.; GUERRA, J. G. M.; ANDRADE, I. P. S.; ROCHA, H. S. Influência da decomposição de diferentes resíduos vegetais submetidos a lâminas de irrigação no comportamento da vegetação espontânea. Acta Scientiarum Agronomy, v. 32, p. 317324, 2010.

TANNER, E. V. J. The decomposition of leaf litter in Jamaican montane rain 
forests. Journal of Ecology, v. 69, p. 263-275, 1981.

THOMAS, R. J.; ASAKAWA, N. M. Decomposition of leaf litter from tropical forage grasses and legumes. Soil Biology and Biochemistry, p. 1351$1361,1993$. 